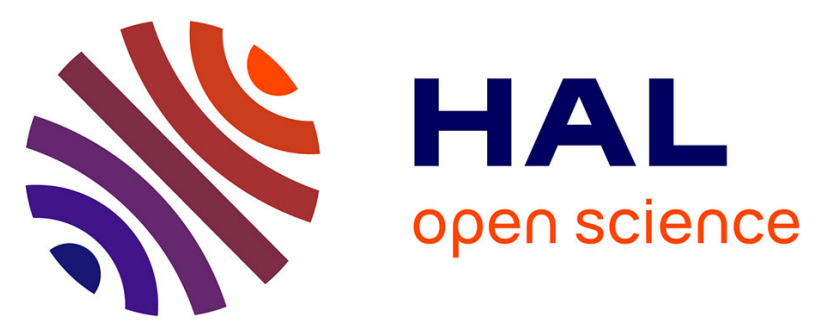

\title{
CLINICAL PATHWAY ANALYSIS USING PROCESS MINING AND DISCRETE-EVENT SIMULATION: AN APPLICATION TO INCISIONAL HERNIA
}

Raksmey Phan, Vincent Augusto, Damien Martin, Marianne Sarazin

\section{- To cite this version:}

Raksmey Phan, Vincent Augusto, Damien Martin, Marianne Sarazin. CLINICAL PATHWAY ANALYSIS USING PROCESS MINING AND DISCRETE-EVENT SIMULATION: AN APPLICATION TO INCISIONAL HERNIA. 2019 Winter Simulation Conference (WSC), Dec 2019, National Harbor, France. pp.1172-1183, 10.1109/WSC40007.2019.9004944 . emse-03128572

HAL Id: emse-03128572

https://hal-emse.ccsd.cnrs.fr/emse-03128572

Submitted on 2 Feb 2021

HAL is a multi-disciplinary open access archive for the deposit and dissemination of scientific research documents, whether they are published or not. The documents may come from teaching and research institutions in France or abroad, or from public or private research centers.
L'archive ouverte pluridisciplinaire HAL, est destinée au dépôt et à la diffusion de documents scientifiques de niveau recherche, publiés ou non, émanant des établissements d'enseignement et de recherche français ou étrangers, des laboratoires publics ou privés. 


\title{
CLINICAL PATHWAY ANALYSIS USING PROCESS MINING AND DISCRETE-EVENT SIMULATION: AN APPLICATION TO INCISIONAL HERNIA
}

\author{
Raksmey Phan \\ Vincent Augusto \\ Center for Biomedical and Healthcare Engineering \\ CNRS, UMR 6158 LIMOS \\ Mines Saint-Étienne \\ Univ. Clermont Auvergne \\ Saint-Étienne, 42023, FRANCE
}

Damien Martin

\author{
Medtronic \\ Sofradim Production \\ 116 avenue du Formans \\ Trevoux, 01600, FRANCE
}

\author{
Marianne Sarazin \\ Institut Pierre Louis d'Epidémiologie et de Santé Publique IPLESP \\ INSERM \\ AP-HP, Hôpital Saint Antoine \\ Sorbonne Université \\ Paris, 75646, FRANCE
}

\begin{abstract}
An incisional hernia (IH) is a ventral hernia that develops after surgical trauma to the abdominal wall, a laparotomy. IH repair is a common surgery that can generate chronic pain, decreased quality of life, and significant healthcare costs caused by hospital readmissions. The goal of this study is to analyze the clinical pathway of patients having an IH using a medico-administrative database. After a preliminary statistical analysis, a process mining approach is proposed to extract the most significant pathways from the database. The resulting causal net is converted into a statechart model that can be simulated. The model is used to understand times of occurrence of complications and associated costs. It enables the simulation of what-if scenarios to propose an improved care pathway for patients who are the most exposed, using in particular prophylactic mesh at the time of abdominal wall closure during a laparotomy on hospitalization costs and readmissions.
\end{abstract}

\section{INTRODUCTION}

The objective of process mining is to use event data to extract process-related information, e.g., to automatically discover a process model by observing events recorded by some enterprise system (Aalst 2011). Two systematic reviews related to process mining were published recently (Maita et al. 2018; Gurgen Erdogan and Tarhan 2018). 705 papers related to process mining published between 2005 and 2014 were gathered in (Maita et al. 2018). For applied studies mentioning the specific application domain, "Medicine and Healthcare" is the second most studied area, and "clinical analysis pathway" is the third type of data sources used for case studies or experiments. A similar analysis was proposed in Gurgen Erdogan and Tarhan 2018 on healthcare studies. 172 papers from 2005 to 2017 have been selected. Process discovery is the most used approach when applying process mining to healthcare, meaning practitioners need more 
knowledge about clinical pathways. It is considered as the most important activity of process mining applied on healthcare. However, there are more studies about "Healthcare process" (93) than "Clinical pathway" (59) meaning organizational problems are still more studied.

Process mining can be combined with discrete optimization (Prodel et al. 2015) and Monte-Carlo simulation (Augusto et al. 2016) to evaluate the performances of new health policies or strategies. In Prodel et al. 2018, a mathematical formulation of the problem has been presented, along with a Tabu Search optimization process to mine the best process model. To reduce the computational time for large-scale data sets, a Monte Carlo sampling method is used. The ICD-10 codes (International Classification of Diseases, 10th revision) has been used for node labeling. In our paper we use a similar approach to mine clinical processes. In Augusto et al. 2016 Monte-Carlo simulation has been used to estimate the impact of new policies regarding medical implants reimbursement. However evaluation and comparison of different clinical pathways with new surgical practices has not been studied.

A case study using both process mining and simulation in health-care is also proposed in Zhou et al. 2014. They used the fuzzy miner algorithm for process discovery (Günther and van der Aalst 2007). They specifically studied the pathway of patients during a single hospital stay, starting from the admission to the release. In our paper, we focus on a macroscopic clinical pathway which can last several years from the first hospital stay to the most recent one.

\section{CASE STUDY AND SCIENTIFIC CONTRIBUTIONS}

\subsection{Case study}

A hernia is a protrusion of tissue or part of an organ through bone, muscular tissue or membrane. More precisely, an incisional hernia (IH) is a ventral hernia that develops after surgical trauma to the abdominal wall, including recurrences after repair of primary ventral hernias. IH repair is a common surgery that can generate chronic pain, decreased quality of life, and significant healthcare costs caused by readmissions. The laparotomy surgeries that may lead to IH are mainly colorectal surgeries, bariatric surgeries, abdominal aortic aneurysm and gynecoligic surgeries. Comorbidities like diabetes, obesity, hypertension and Chronic Obstructive Pulmonary Disease (COPD) have been identified as main risk factors.

Among patients having a laparotomy, $10 \%$ to $25 \%$ will develop an incisional hernia (Deerenberg 2017). The ratio can be even higher, depending on the type of surgery, risk factors, suture material and surgical technique. The reported incidence is determined by several factors: (i) patient population, (ii) type of abdominal wall incision, (iii) length of follow-up, and (iv) method of IH diagnosis (Muysoms et al. 2015). For example, a midline incision due to an abdominal cancer operation is strongly associated with hernia formation, IH occurred for $41 \%$ of patients (Baucom et al. 2016).

However even small incisions due to a laparoscopic surgery can generate IH. The incidence of IH after midline and off-midline incisions in patients undergoing laparoscopic colorectal surgery showed that the pooled incidence of $\mathrm{IH}$ was $10.6 \%$ in midline, $3.7 \%$ in transverse, and $0.9 \%$ in Pfannenstiel incisions (Lee et al. 2017). After an IH repair, the recurrence rate is above $33 \%$, but with a re-surgery the rate is lower (Helgstrand et al. 2012). A patient is also likely to have a surgical site occurrence like a surgical site infection (SSI) or a seroma. The risk of SSI depends of the type of IH repair, raising from $3.4 \%$ for a laparoscopic repair to $22.1 \%$ for an open IH repair (Kaafarani et al. 2010), and factors like obesity, COPD or the porosity of the mesh have a significant impact (Sanchez et al. 2011).

Decrease of quality of life of patients is not the only issue. Costs of IH repair are a burden from the societal point of view. The mean total cost for an IH repair was estimated to be 6,451 euro ranging from 4,731 euro to 10,107 euro for respectively unemployed and employed patients (Gillion et al. 2016). Also higher costs occur when the IH appears within 1 year versus 3 years (Alli et al. 2018).

Prophylactic mesh are promising implants that can be used during laparotomy to prevent IH (Hernando et al. 2016). Such medical act is safe and effectively prevents the development of IH for at least two years for abdominal aortic aneurysm (Muysoms et al. 2016). It is even proposed as a standard of care for 
patients at high risk (Muysoms and Dietz 2017). However practitioners need guidelines to find out which patients should benefit such implant.

\subsection{Objectives and scientific contributions}

The objective of this study is twofold:

1. Propose an analysis framework based on process mining and discrete-event simulation using medicoadministrative data to evaluate what-if scenarios of clinical pathways such as public health strategies.

2. Provide quantitative results on a case study related to IH patients clinical pathway.

The main scientific challenge of this study lies in the definition and development of the analysis framework, especially regarding the process mining causal net conversion into a discrete-event simulation model. From a medical point of view, what-if scenarios should be carefully tuned in order to get realistic results that can be used to develop new public health strategies such as new medical act reimbursement.

The remainder of this paper is organized as follows. A short literature review on clinical pathway modeling and simulation is presented in Section 1. The position of the problem is given in Section 3. A data description is provided in Section 4. The process mining approach is detailed in Section 5 along with the discrete-event simulation model generation. Numerical results on the IH case study are given in Section 6. Conclusions and perspectives are discussed in Section 7.

\section{OVERVIEW OF THE ANALYSIS FRAMEWORK}

In this paper we intend to propose an analysis framework based on process mining and discrete-event simulation using medico-administrative data to evaluate what-if scenarios of clinical pathways such as public health strategies. Such framework will be evaluated on a case study related to incisional hernia.

Our methodology is illustrated in Figure 1. Data is collected from medico-administrative database, as presented in Section 4. Data Extraction (step 1) consists of querying the database. At this step, we also validate the cohort with health practitioners using statistical analysis. We then convert the original data into event logs in order to be able to apply process mining (step 2). In this second step, we use existing mining algorithms such as extensions of Fuzzy Miner (Günther and van der Aalst 2007) on which the Disco software is based. Finally, the model is converted again into a discrete-event simulation model in order to perform what-if scenarios simulations of new clinical pathway strategies (step 3).

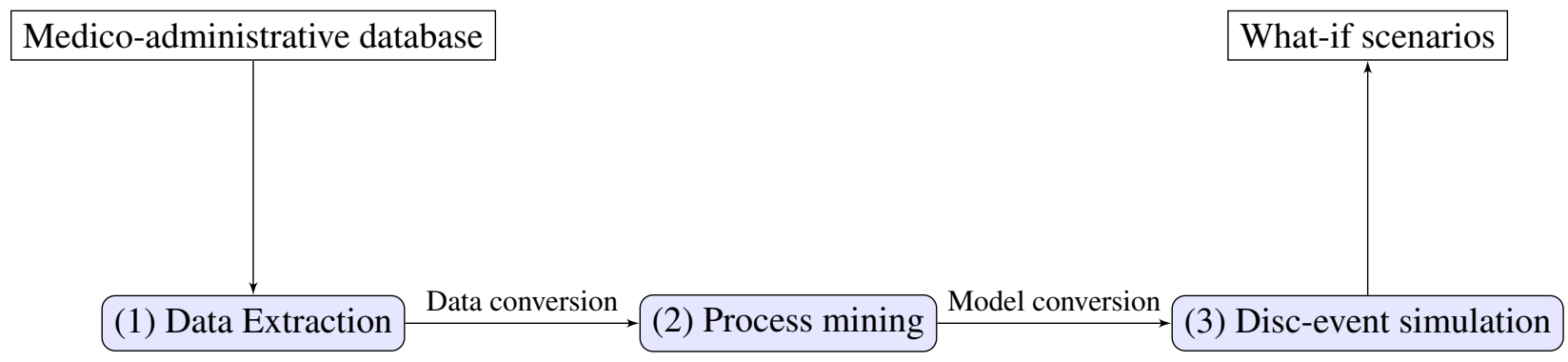

Figure 1: Global methodology.

The following sections of this paper present the medico-administrative data used for this study (Section 4). Then the process mining approach and the simulation model generation are described in Section 5. The performance of the approach is evaluated on a real case study related to clinical pathway of patients having incisional hernia in Section 6. 


\section{DATA DESCRIPTION}

Data is extracted from the French national hospital discharge database (PMSI: "Programme de Médicalisation des Systèmes d'Information") using the International Classification of Diseases (ICD-10) and the French classification called "Common Classification of Medical Acts" (Classification Commune des Actes Médicaux in French, CCAM 2010). Surgeries done by laparotomy represent 431,719 patients in 2010 . There are $71 \%$ of females and the average age is 42 , due to the high number of caesarean section into this population. Among these patients, 22,088 (5\%) have had a first incisional hernia (IH) in the following years. Patients having at least one $\mathrm{IH}$ are around 62 years old and are in majority women (55\%). Figure 2 summarizes this first statistical analysis.

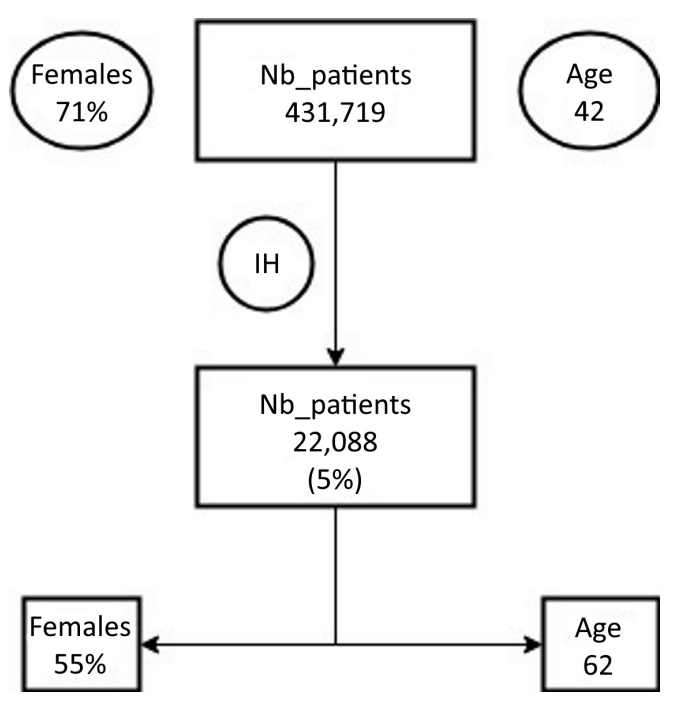

Figure 2: Extraction of 22,088 patients with IH from 431,719 patients with laparotomy.

According to the ICD-10, there are about 556 codes related to the laparotomy surgical operations. In this paper we focus only on laparotomies generating the highest volumes of $\mathrm{IH}$ according to two criteria.

1. The first criterion is the number of patients: we selected laparotopy surgeries performed on at least 100 patients. Such selection allows to avoid irrelevant surgeries for this study.

2. The second criterion is the rate of IH: we decided to select only laparotomy surgeries that lead to at least $10 \%$ of occurrence rate of $\mathrm{IH}$; such decision allows to avoid surgeries representing a high number of patients but with a low incidence rate of IH. For example, surgeries like "Scheduled cesarean section by laparotomy" having 59,000 patients with an IH occurrence rate of only $0.9 \%$ are put aside.

As such, all the following statistics and analysis will be more accurate and not diluted. With this selection the number of studied laparotomy codes has been reduced to 65 (top-65). This top-65 represents 71,863 patients with an average rate of incisional hernia in the next 5 years of $17 \%$.

Patients having an IH are twice more likely to have at least one comorbidity factor in comparison to someone without an IH. On the top-65, a study of the impact of the 4 selected comorbidity factors has been done: Chronic Obstructive Pulmonary Diseases (COPD), Diabetes, Hypertension and Obesity. Results are displayed in Table 1. It shows that patients with at least one of those comorbidities are more likely to have $\mathrm{IH}$ in the next 5 years (obesity seems to be the most impactful factor). 
Table 1: Rate of incisional hernia dependent on comorbidities.

\begin{tabular}{|l|ll|ll|ll|ll|}
\hline Factor & \multicolumn{2}{|c|}{ COPD } & \multicolumn{2}{c|}{ Diabetes } & \multicolumn{2}{c|}{ Hypertension } & \multicolumn{2}{c|}{ Obesity } \\
& no & yes & no & yes & no & yes & no & yes \\
\hline \#patients & 67,131 & 4,732 & 61,386 & 10,477 & 41,287 & 30,576 & 63,131 & 8,732 \\
IH_rate & $16 \%$ & $20 \%$ & $16 \%$ & $19 \%$ & $15 \%$ & $19 \%$ & $15 \%$ & $31 \%$ \\
\hline
\end{tabular}

\section{CLINICAL PATHWAY PROCESS MINING AND SIMULATION}

This section introduces the process mining approach used in this paper. The process model and the preprocessing data step will be presented. The readers are referred to Aalst 2011, Günther 2009 and Augusto et al. 2016 for more details on the basics of process mining including formal definitions of concepts of events, traces, and logs.

\subsection{Data conversion for process mining}

A data conversion procedure is necessary before applying process mining to medico-administrative data. The objective of this procedure is to obtain an event $\log$, the basic material to apply any process mining algorithm. Contrary to Augusto et al. 2016 where event labels are Diagnosis Related Groups (DRG), important information to define nodes of our process model are contained in medical acts performed during a patient stay in the hospital. Thus we need to extract both Diagnosis Related Groups (DRG) that characterize a hospital stay, as well as medical acts performed during a hospital stay. To extract such information, we need to duplicate a hospital stay into several log entries.

Figure 3 shows an example of this conversion step: for Patient A who had 3 medical acts during a hospital stay labeled "IH", we generate 3 events in the log.

\begin{tabular}{|c|c|c|c|}
\hline Patient id & Date & DRG & Medical acts \\
\hline Patient A & Date of stay & IH & Incisional hernia repair; Mesh ablation; Blood infusion \\
\hline \multicolumn{3}{|c}{} \\
$\downarrow$
\end{tabular}

\begin{tabular}{|c|c|c|}
\hline Patient id & Date & Event \\
\hline Patient A & Date of incisional hernia repair & Incisional hernia repair \\
Patient A & Date of mesh ablation & Mesh ablation \\
Patient A & Date of blood infusion & Blood infusion \\
\hline
\end{tabular}

Figure 3: Conversion of a stay into 3 surgeries.

Then it is necessary to focus on one medical act that is incisional hernia repair in the clinical pathway of patients. It is the inclusion point of our patients and events before and after this central event should be distinguished. An example is proposed in Figure 4 where a patient has a blood infusion before and after an incisional hernia that should be discriminated.

\subsection{Process mining and event refinement}

Using the converted data, we are able to apply any process mining algorithm. In our case we use Disco ${ }^{1}$, a process mining tool to generate graph in which each node contains the CCAM code of the surgery whereas the frequencies are displayed for each node and arc.

\footnotetext{
${ }^{1}$ Fluxicon - DISCOver your processes: https://fluxicon.com/disco/
} 
Phan, Martin, Augusto, and Sarazin

\begin{tabular}{|c|c|c|}
\hline Patient id & Date & Event \\
\hline Patient A & Date of blood infusion & Blood infusion \\
Patient A & Date of incisional hernia repair & Incisional hernia repair \\
Patient A & Date of blood infusion & Blood infusion \\
\hline \multicolumn{2}{|c}{} \\
$\downarrow$
\end{tabular}

\begin{tabular}{|c|c|c|}
\hline Patient id & Date & Event \\
\hline Patient A & Date of blood infusion & Blood infusion before \\
Patient A & Date of incisional hernia repair & Incisional hernia repair \\
Patient A & Date of blood infusion & Blood infusion after \\
\hline
\end{tabular}

Figure 4: Disctinction between events before and after an IH.

The next step is to define medical acts that are relevant regarding incisional hernia occurrence analysis. For example it is not relevant to know that 2 months before a laparotomy a patient had an ossiculoplasty (an operation to repair, reconstruct and improve the movement of the tiny bones of hearing in the middle ear). Based on practitioners expertise, we focus only on the CCAM codes given by Table 2 .

Table 2: Incisional hernia repair codes in the CCAM.

\begin{tabular}{|r|l|}
\hline CCAM-Code & Description \\
\hline LMMA004 & Cure of postoperative IH of the anterior abdominal wall w/ mesh, by direct approach \\
LMMA010 & Cure of postoperative IH of the anterior abdominal wall w/o mesh, by direct approach \\
LMMC015 & Cure of postoperative IH of the anterior abdominal wall w/ mesh, by laparoscopy \\
\hline
\end{tabular}

Due to space limitation, we focus in this paper on the analysis of three clinical pathways regarding IH recurrence and occurrence: (i) for general patients (Figure 5), and (ii) for non-obese patients and for obese patients (Figure 6).

\subsection{Discrete-event simulation model generation}

To generate the simulation model, we use a state chart conversion procedure as defined in Augusto et al. 2016. This is a step by step conversion procedure in order to produce a simulation model using as input a causal net generated using a process mining approach such as Fuzzy Miner. The input material of our procedure is a process model. Such model is denoted Clinical Pathway State Chart and is defined as follows.

Definition 1 (CP State Chart) A Clinical Pathway State Chart (CPSC) is a state chart $M$ which underlying graph is a bigraph (bipartite graph) having the following properties:

- The two types of states of the bipartite graph are Care-states and Wait-states. By definition, two states of the same type cannot be linked by a transition.

- Each Wait-state has exactly one input transition and one output transition: the probability of activating the output transition of a Wait-state is always equal to 1 .

- The sum of all probabilities of output transitions of a Care-state is equal to 1 .

According to Definition 1, a Care-state may be followed by $\{0 ; n\}$ Wait-state(s) whereas a Wait-state is always followed by exactly one Care-state: the probability of the transition between a Wait-state and a Care-state is equal to 1.

The resulting simulation model has been implemented using Anylogic and is illustrated in Figure 7. Parameters and scenarios are detailed in the following Section. 
Phan, Martin, Augusto, and Sarazin

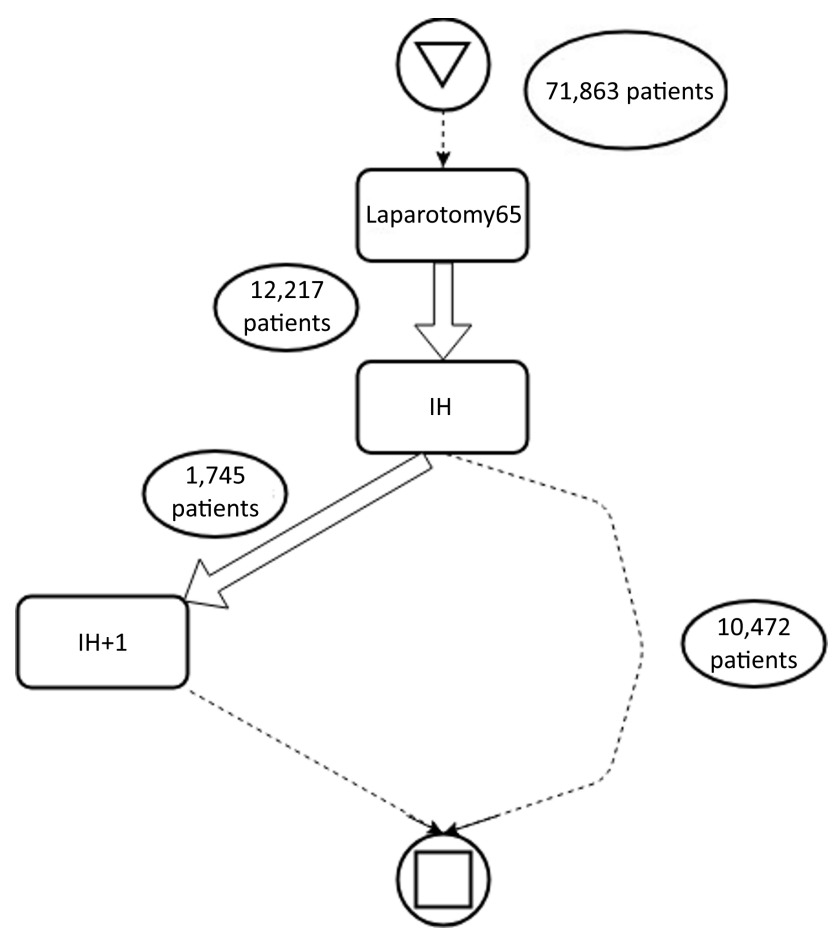

Figure 5: Process mining on general patients population.
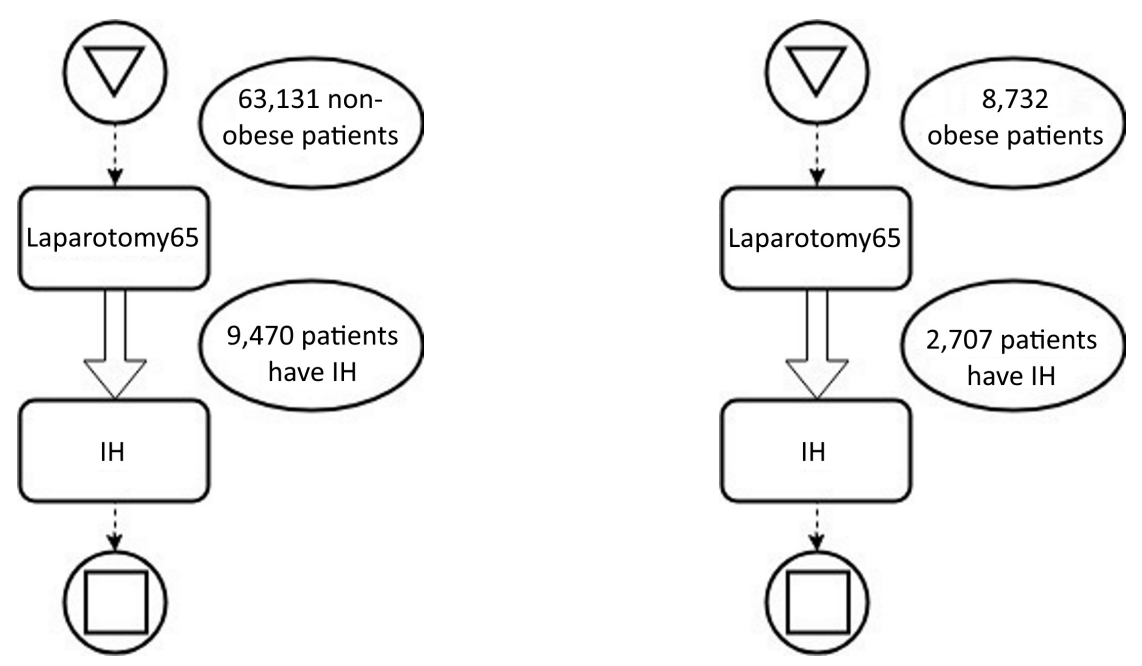

Figure 6: Process mining on non obese (left) and obese (right) patients.

\section{NUMERICAL RESULTS}

The goal of the simulation is to evaluate the economic impact of using a prophylactic mesh after a laparotomy to prevent incisional hernia. Clinical studies are currently done and seem to be encouraging. In this paper we focus on the following questions:

- Does using a prophylactic mesh after a laparotomy shorten the clinical pathway of patients?

- Are hospitals and healthcare systems going to save money thanks to the use of a prophylactic mesh at the time of initial laparotomy closure? 


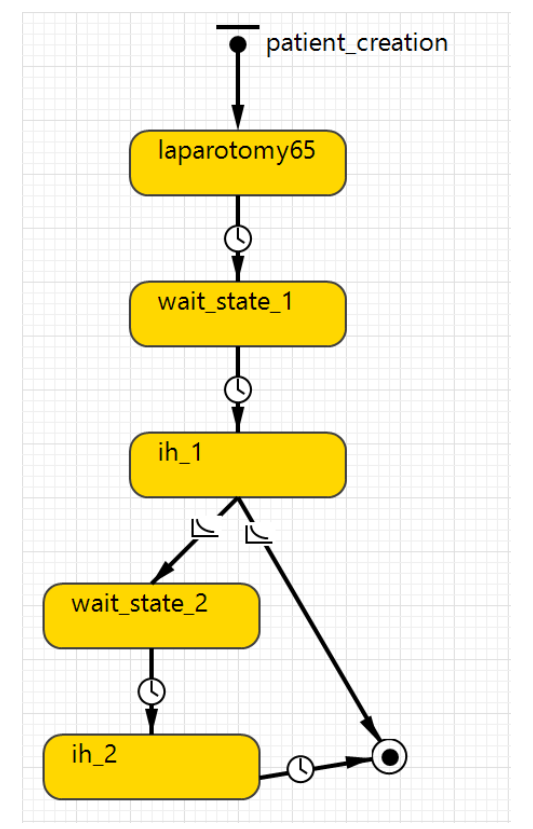

Figure 7: Resulting simulation model.

\subsection{Design of experiments}

The simulation has been done with Anylogic simulation software. Below is shown a scheme of the simulation. It takes into account only patients with a laparotomy in the top-65 of the previous data analysis. Possible events for those patients are:

- Use of a prophylactic mesh.

- Incisional hernia occurrence.

- Incisional hernia recurrence.

The simulation also takes into account the difference between obese and non-obese patients. The most critical post-operative complication, the risk of having a mesh ablation after the use of a prophylactic mesh and after an incisional hernia occurrence is also considered; note that other complications can occur after the use of mesh but the lack of information about these complications the associated costs in particular have limited our capacities to take them into consideration in this evaluation.

The two main key performance indicators are: (1) The average direct cost for one patient after the laparotomy until a potential incisional hernia recurrence; and (2) the average number of hospital stays for one patient after a laparotomy until a potential incisional hernia recurrence.

Figure 8 summarizes the simulation scheme. We simulated 72,000 patients (from 2010) having a laparotomy among the top-65, with Mesh ablation rate of 3\% and obesity rate of $13 \%$ (obtained from data analysis). We compare population with prophylactic mesh and without.

\subsection{Data}

Information used for these simulations come from previous data analysis and hospital reimbursement data. Only occurrence rates for population with prophylactic mesh are extracted from recent articles (Hernando et al. 2016; Muysoms et al. 2016; Muysoms and Dietz 2017). Table 3 gives the incisional hernia occurence and recurrence rates for obese and non-obese patients dependent on whether they have (or not) a prophylactic 


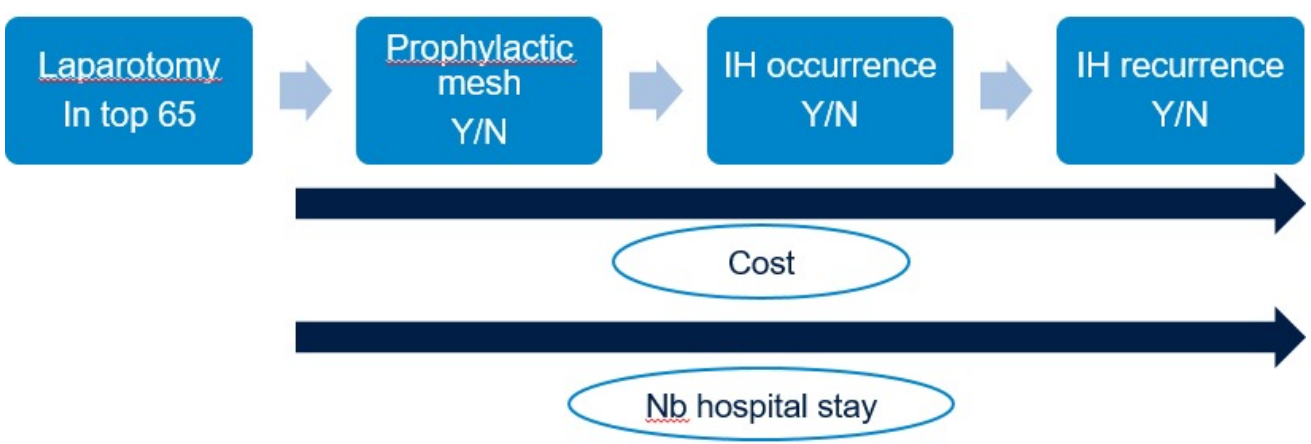

The simulation start with a patient having a laparotomy in the top 65 and end with a possible $\mathrm{IH}$ recurrence

Figure 8: Simulation scheme.

mesh. Of course, all patients that have incisional hernia recurrence also have a prophylactic mesh. We can notice that the occurrence rate for patients with prophylactic mesh is very small. Table 4 gives the cost value for each type of surgical operation (incisional hernia repair, incisional recurrent hernia repair, prophylactic mesh and mesh ablation) dependent on patients with or without obesity. Note that the cost value for prophylactic mesh is given by Medtronic.

Table 3: Incisional hernia rate depending on prophylactic mesh.

\begin{tabular}{|r|rr|rr|}
\hline & \multicolumn{2}{|c|}{ Occurrence } & \multicolumn{2}{c|}{ Recurrence } \\
\hline Prophylactic mesh & No obesity & Obesity & No obesity & Obesity \\
no & $15 \%$ & $31 \%$ & - & - \\
yes & $3 \%$ & $5 \%$ & $13 \%$ & $19 \%$ \\
\hline
\end{tabular}

Table 4: Surgical operation cost value depending on patients obesity.

\begin{tabular}{|r|rr|}
\hline & No obesity & Obesity \\
\hline Incisional hernia repair & $1,972 €$ & $10,894 €$ \\
Incisional hernia recurrence repair & $3,333 €$ & $18,411 €$ \\
Prophylactic mesh & $200 €$ & $200 €$ \\
Mesh ablation & $2,320 €$ & $18,088 €$ \\
\hline
\end{tabular}

\subsection{Results}

Simulations have been done to analyse results about general population, to compare obese and non-obese patients and to evaluate the total cost for each case. In the following, column "Prophylactic Mesh" presents the two scenarios: with and without mesh; column "\#stay" gives the average number of times that patients come to the hospital for mesh ablation, incisional hernia occurrence or incisional hernia recurrence and thus should be as small as possible; and finally column "Cost by patient" gives the average cost value for each patient.

Table 5 shows that for the general population, the number of stays is divided by 2.7 when comparing population without mesh to population with mesh. The average direct cost for each patient is also decreased by $45 \%$ by using a prophylactic mesh for every patient. These results show the importance to consider the use of prophylactic mesh on the general population.

Since obesity increases the risk of incisional hernia, Table 6 gives results regarding the same analysis on obese and non-obese population. In this case, we consider that a non-obese patient is always associated 
Table 5: Table of simulation for the General Population.

\begin{tabular}{|r|ll|}
\hline Prophylactic Mesh & \#stay & Cost/patient \\
\hline no & 0.2 & $926 €$ \\
yes & 0.07 & $512 €$ \\
\hline
\end{tabular}

with the lowest cost at each stage of his medical pathway, whereas an obese patient is always associated with the highest cost at each stage of his medical pathway (note that the highest cost in the medical pathway is usually associated to morbidly obese patients). As it is shown in Table 6, for non-obese patients the use of a prophylactic mesh still decreases largely the average number of times that patients come to the hospital (\#stay), even if the average cost value for each patient (Cost/patient) is similar. Regarding the obese population, the amount of stays (\#stay) is divided by 4 when a prophylactic mesh is used and the average cost value is decreased by $65 \%$. In addition to the previous analysis about general population, these results highlight the benefit of using prophylactic mesh for obese patients.

Table 6: Number of stays and cost per patient.

\begin{tabular}{|r|ll|ll|}
\hline & \multicolumn{2}{|c|}{ Non-obese } & \multicolumn{2}{c|}{ Obese } \\
\hline Prophylactic Mesh & \#stay & Cost/patient & \#stay & Cost/patient \\
\hline no & 0.17 & $369 €$ & 0.38 & $4,621 €$ \\
yes & 0.07 & $348 €$ & 0.10 & $1,595 €$ \\
\hline
\end{tabular}

A cost analysis is finally provided. Table 7 presents the total cost for general population (column "total"), for non-obese (column "Non-obese") and obese (column "Obese") population. Results show that the main savings come from the prevention of incisional hernia for obese patients. Indeed, the cost savings for non-obese patients represent only $7 \%$ of the savings whereas there make up $87 \%$ of the population.

Table 7: Costs evaluation.

\begin{tabular}{|r|lll|}
\hline Prophylactic Mesh & Total & Non-obese & Obese \\
\hline no & 66 million $€$ & 23 million $€$ & 43 million $€$ \\
yes & 36 million $€$ & 21 million $€$ & 15 million $€$ \\
save & 30 million $€$ & 2 million $€$ & 28 million $€$ \\
\hline
\end{tabular}

\section{CONCLUSIONS AND PERSPECTIVES}

Process Mining is an observed behaviour approach that uses event-log data to describe "what really happened"; so it leads to realistic simulation models. With this approach, it is easy to have a model that generates events for statistical analysis. Such analysis (combining process mining and simulation) shows that the use of prophylaxis mesh significantly decreases the cost of medical care and enhances the clinical pathway (by reducing the average number of times that patients come to the hospital) after a laparotomy and particularly for obese patients. However these simulations have multiple limitations. Indeed, (1) it does not consider post operative complications like seroma or surgical site infection; (2) in case of multi operations by laparotomy, it is supposed that the surgery site is always the same; and (3) the previous laparotomy before the IH is selected however it could come from an anterior surgery. Also we could not take into account the impact of the experience of the surgeon and the type of closure after the laparotomy.

The proposed methodology has proven effective and brings new insight for practitioners. However, the method is difficult to generalize to other data sources or case studies because of the special features related to each medical case. For future works, we intend to develop a generic data extraction for process mining and simulation that could be embedded in a software. 
Phan, Martin, Augusto, and Sarazin

\section{ACKNOWLEDGMENTS}

Authors would like to thank Mrs. Linda Spinnler and Mr. Philippe Gravagna from the Medtronic group (Sofradim Production) who supported this study.

\section{REFERENCES}

Aalst, W. M. P. 2011. Process Mining: Discovery, Conformance and Enhancement of Business Processes. Berlin: Springer-Verlag.

Alli, V. V., J. Zhang, and D. A. Telem. 2018. "Impact of Incisional Hernia Development Following Abdominal Operations on Total Healthcare Cost”. Surgical Endoscopy 32(5):2381-2386.

Augusto, V., X. Xie, M. Prodel, B. Jouaneton, and L. Lamarsalle. 2016. "Evaluation of Discovered Clinical Pathways using Process Mining and Joint Agent-Based Discrete-Event Simulation". In Proceedings of the 2016 Winter Simulation Conference, edited by P. Frazier, T. M. Roeder, R. Szechtman, and E. Zhou, 2135-2146. Piscataway, New Jersey: Institute of Electrical and Electronics Engineers, Inc.

Baucom, R. B., J. Ousley, G. B. Beveridge, S. E. Phillips, R. A. Pierce, M. D. Holzman, K. W. Sharp, W. H. Nealon, and B. K. Poulose. 2016. "Cancer Survivorship: Defining the Incidence of Incisional Hernia After Resection for Intra-Abdominal Malignancy”. Annals of Surgical Oncology 23(5):764-771.

Deerenberg, E. 2017. Prevention and Treatment of Incisional Hernia: New Techniques and Materials. Ph.D. thesis, Erasmus University Rotterdam, Rotterdam, Netherlands. http://hdl.handle.net/1765/98646, accessed 20 ${ }^{\text {th }}$ August 2019.

Gillion, J.-F., D. Sanders, M. Miserez, and F. Muysoms. 2016. "The Economic Burden of Incisional Ventral Hernia Repair: A Multicentric Cost Analysis". Hernia 20(6):819-830.

Günther, C. W. 2009. Process Mining in Flexible Environments. Ph.D. thesis, Eindhoven University of Technology, Eindhoven, Netherlands. http://www.processmining.org/blogs/pub2009/process_mining_in_flexible_environments, accessed 20 ${ }^{\text {th }}$ August 2019.

Günther, C. W., and W. M. P. van der Aalst. 2007. "Fuzzy Mining - Adaptive Process Simplification Based on Multi-perspective Metrics". In Proceedings of Business Process Management, edited by G. Alonso, P. Dadam, and M. Rosemann, 328-343. Berlin: Springer-Verlag.

Gurgen Erdogan, T., and A. Tarhan. 2018. "Systematic Mapping of Process Mining Studies in Healthcare”. IEEE Access 6:1-1.

Helgstrand, F., J. Rosenberg, H. Kehlet, P. Strandfelt, and T. Bisgaard. 2012. "Reoperation Versus Clinical Recurrence Rate After Ventral Hernia Repair”. Annals of Surgery 256(6):955-958.

Hernando, L. A. B., M. ngel Garca-Urea, J. Lpez-Moncls, S. G. Hernndez, lvaro Robn Valle de Lersundi, A. C. Cidoncha, D. M. Montes, C. C. Pavn, E. G. Gonzlez, and N. P. Garca. 2016. "Prophylactic Mesh can be used Safely in the Prevention of Incisional Hernia after Bilateral Subcostal Laparotomies”. Surgery 160(5):1358 - 1366.

Kaafarani, H. M., D. Kaufman, D. Reda, and K. M. Itani. 2010. "Predictors of Surgical Site Infection in Laparoscopic and Open Ventral Incisional Herniorrhaphy”. Journal of Surgical Research 163(2):229 - 234.

Lee, L., M. Abou-Khalil, S. Liberman, M. Boutros, G. M. Fried, and L. S. Feldman. 2017. "Incidence of Incisional Hernia in the Specimen Extraction Site for Laparoscopic Colorectal Surgery: Systematic Review and Meta-Analysis". Surgical Endoscopy 31(12):5083-5093.

Maita, A. R. C., L. C. Martins, C. R. L. Paz, L. Rafferty, P. C. K. Hung, S. M. Peres, and M. Fantinato. 2018. "A Systematic Mapping Study of Process Mining". Enterprise Information Systems 12(5):505-549.

Muysoms, F. E., S. A. Antoniou, K. Bury, G. Campanelli, J. Conze, D. Cuccurullo, A. C. de Beaux, E. B. Deerenberg, B. East, R. H. Fortelny, J.-F. Gillion, N. A. Henriksen, L. Israelsson, A. Jairam, A. Jänes, J. Jeekel, M. López-Cano, M. Miserez, S. Morales-Conde, D. L. Sanders, M. P. Simons, M. Śmietański, L. Venclauskas, and F. Berrevoet. 2015. "European Hernia Society Guidelines on the Closure of Abdominal Wall Incisions". Hernia 19(1):1-24.

Muysoms, F. E., O. Detry, T. Vierendeels, M. Huyghe, M. Miserez, M. Ruppert, T. Tollens, J.-O. Defraigne, and F. Berrevoet. 2016. "Prevention of Incisional Hernias by Prophylactic Mesh-augmented Reinforcement of Midline Laparotomies for Abdominal Aortic Aneurysm Treatment: A Randomized Controlled Trial". Annals of Surgery 263:638-645.

Muysoms, F. E., and U. A. Dietz. 2017. "Prophylactic Meshes in the Abdominal Wall". Der Chirurg 88(1):34-41.

Prodel, M., V. Augusto, B. Jouaneton, L. Lamarsalle, and X. Xie. 2018. "Optimal Process Mining for Large and Complex Event Logs". IEEE Transactions on Automation Science and Engineering 15(3):1309-1325.

Prodel, M., V. Augusto, X. Xie, B. Jouaneton, and L. Lamarsalle. 2015. "Discovery of Patient Pathways from a National Hospital Database using Process Mining and Integer Linear Programming”. In Proceedings of the 2015 International Conference on Automation Science and Engineering, edited by Q. S. Jia, M. P. Fanti, D. Popa, and C. Seatzu, 1409-1414. Piscataway, New Jersey: Institute of Electrical and Electronics Engineers, Inc.

Sanchez, V. M., Y. E. Abi-Haidar, and K. M. Itani. 2011. "Mesh Infection in Ventral Incisional Hernia Repair: Incidence, Contributing Factors, and Treatment”. Surgical Infections 12(3):205-210. 
Zhou, Z., Y. Wang, and L. Li. 2014. "Process Mining Based Modeling and Analysis of Workflows in Clinical Care - A Case Study in a Chicago Outpatient Clinic". In Proceedings of the 11th International Conference on Networking, Sensing and Control, 590-595. Piscataway, New Jersey: Institute of Electrical and Electronics Engineers, Inc.

\section{AUTHOR BIOGRAPHIES}

RAKSMEY PHAN received his Ph.D. degree from the Institut Suprieur d'Informatique, de Modlisation et de leurs Applications (ISIMA), France, in 2013 and his engineering degree, in 2010. Currently, he is a researcher-engineer in the Center for Health Engineering and in the IEOR team of CNRS UMR 6158 LIMOS, EMSE. His fields of interest include Operational Research, Databases and Internet of Things. His e-mail and web addresses are raksmey.phan@ mines-stetienne.fr and http://emse.raksmey.fr/, respectively.

DAMIEN MARTIN received his engineer degree (equivalent to a Master of Science) from IMT Mines Albi-Carmaux, France in 2019. His email address is damien.martin@mines-albi.fr.

VINCENT AUGUSTO is a Professor of industrial engineering in the Center for Health Care Engineering at Mines SaintEtienne. He received his Ph.D. in Industrial Engineering from Mines Saint-Étienne, France, in 2008 and the Habilitation à Diriger des Recherches degree from the University of Saint-Étienne, France, in 2016. He was a visiting scholar at CIRRELT (Centre Interuniversitaire de Recherche sur les Réseaux d'Entreprise, la Logistique et le Transport), University of Laval, Quebec, Canada in 2009 and 2015. His research interests include modeling, simulation, optimization of healthcare systems and their supply chains, process mining and machine learning applied to healthcare. His email address is augusto@emse.fr.

MARIANNE SARAZIN is a public health doctor with a doctorate in Life Sciences from Mines Saint-Étienne, France. She is the head of the Medical Information Department of the Mutualiste Sanitary Group of Saint-Étienne and a collaborator in the Center for Health Care Engineering at Mines Saint-Etienne. She is also with the UMRS 1136 Inserm lab, specialized in the modeling of epidemics. Her email address is marianne.sarazin@iplesp.upmc.fr. 